\title{
ON THE GAUSSIAN BEHAVIOR OF MARGINALS AND THE MEAN WIDTH OF RANDOM POLYTOPES
}

\author{
DAVID ALONSO-GUTIÉRREZ AND JOSCHA PROCHNO
}

(Communicated by David Levin)

\begin{abstract}
We show that the expected value of the mean width of a random polytope generated by $N$ random vectors $\left(n \leq N \leq e^{\sqrt{n}}\right)$ uniformly distributed in an isotropic convex body in $\mathbb{R}^{n}$ is of the order $\sqrt{\log N} L_{K}$. This completes a result of Dafnis, Giannopoulos and Tsolomitis. We also prove some results in connection with the 1-dimensional marginals of the uniform probability measure on an isotropic convex body, extending the interval in which the average of the distribution functions of those marginals behaves in a sub- or supergaussian way.
\end{abstract}

\section{INTRODUCTION}

In asymptotic convex geometry, the hyperplane conjecture is a very well-known problem that first appeared explicitly in [9]. This conjecture says that there exists an absolute constant $c$ such that every convex body of volume 1 has a hyperplane section of volume greater than $c$. A result by Hensley [16] yields an equivalent formulation, saying that there exists an absolute constant $c$ such that every convex body has isotropic constant $L_{K}$ less than $c$.

The study of random polytopes began with Sylvester and the famous four-point problem nearly 150 years ago. Since then, a tremendous effort has been made to study expectations, variances, and distributions of several functionals on a random polytope. This turned out to be very useful and many applications have been found (see [3], 32] and references therein). Random polytopes also provided counterexamples to several conjectures (see, for instance, [14, 34, 15] or [23]).

In 1989, Milman and Pajor 22] showed a deep connection between the hyperplane conjecture and the study of random polytopes, proving that the expected volume of a random simplex in an isotropic convex body is closely related to the value of its isotropic constant.

In [12, the authors studied the expected value of the quermaßintegrals of a random polytope generated by $N$ random vectors uniformly distributed in an isotropic convex body in $\mathbb{R}^{n}$. They showed that if $n \leq N \leq e^{\sqrt{n}}$, then the expected value of the smallest quermaßintegral, which is the volume radius, is greater than $c \sqrt{\log \frac{N}{n}} L_{K}$ and the expected value of the biggest one, which is the mean width, is smaller than $C \sqrt{\log N} L_{K}$. Actually, the upper estimate had been proved in [1]. A similar lower bound without $L_{K}$ had also been proved there. This yields a sharp

Received by the editors May 28, 2012 and, in revised form, March 22, 2013.

2010 Mathematics Subject Classification. Primary 52A22; Secondary 52A23, 05D40, 46B09.

Key words and phrases. Supergaussian direction, random polytope, Orlicz norm, mean width. 
estimate for the expected value of any quermaßintegral when $n^{2} \leq N \leq e^{\sqrt{n}}$, but leaves a gap for the range $n \leq N \leq n^{2}$. Our first purpose is to fill this gap for the expected value of the mean width. Denoting by $a \sim b$ the fact that there exist positive absolute constants $c, C$ such that $c a \leq b \leq C a$, in Section 3 we will prove the following:

Theorem 1.1. Let $K \subseteq \mathbb{R}^{n}$ be a symmetric isotropic convex body and $n \leq N \leq$ $e^{\sqrt{n}}$. Let $K_{N}=\operatorname{conv}\left\{ \pm X_{1}, \ldots, \pm X_{N}\right\}$ be a random polytope, where $X_{1}, \ldots, X_{N}$ are independent random vectors uniformly distributed in $K$. Then,

$$
\mathbb{E} w\left(K_{N}\right) \sim \sqrt{\log N} L_{K} .
$$

This estimate in the range $n \leq N \leq n^{2}$ will be a consequence of the central limit theorem for convex bodies proved by Klartag [19] and the results proved by Sodin [33]. The central limit theorem for convex bodies was first considered in [2] and says that most of the 1-dimensional marginals $\langle X, \theta\rangle$ of a random variable $X$ uniformly distributed in an isotropic convex body $K$ are, in a certain sense, approximately Gaussian. To be more precise, Klartag showed that the distribution function $F_{\theta}(t)$ of most of these marginals is "almost" Gaussian whenever $|t|$ is smaller than some power of $n$. It turns out that the Gaussian behavior for a particular value of $t$ in this range will be enough to prove Theorem 1.1.

Along these lines a great deal of research was devoted in connection with the marginals of the uniform probability measure on an isotropic convex body and important results were obtained. For instance, in [10] Bourgain verified the hyperplane conjecture for the class of $\psi_{2}$ bodies, i.e., the class of convex bodies such that every direction (or 1-dimensional marginal) is subgaussian. Given an isotropic convex body $K$, we say that a direction $\theta \in S^{n-1}$ is subgaussian with constant $r>0$ if

for all $1 \leq t \leq r \sqrt{n}$.

$$
\left|\left\{x \in K:|\langle x, \theta\rangle| \geq t L_{K}\right\}\right| \leq e^{-\frac{t^{2}}{r^{2}}}
$$

In this setting, the following question was posed by Milman: is it true that every convex body has at least one subgaussian direction? This question has been answered in the affirmative for the class of 1-unconditional convex bodies [8], for the class of zonoids 26, and for the class of isotropic convex bodies with small diameter [27]. (In fact, it was shown that the measure of subgaussian directions is greater than $1-e^{-\sqrt{n}}$ for this last class of convex bodies.) In 18, Klartag established the existence of a subgaussian direction up to a logarithmic factor in the dimension. See also 13 .

In 30, Pivovarov considered the dual question of finding supergaussian directions. We say that a direction $\theta \in S^{n-1}$ is supergaussian with constant $r>0$ if for all $1 \leq t \leq \frac{\sqrt{n}}{r}$ we have

$$
\left|\left\{x \in K:|\langle x, \theta\rangle| \geq t L_{K}\right\}\right| \geq e^{-r^{2} t^{2}} .
$$

He gave an affirmative answer up to a logarithmic factor for the class of 1-unconditional convex bodies.

In [28, Paouris showed that every isotropic convex body with bounded isotropic constant has "many" supergaussian directions. This includes several classes of convex bodies such as 1-unconditional convex bodies, zonoids, duals of zonoids, and the unit balls of the Schatten classes. He also proved that if for every isotropic 
convex body a random direction is supergaussian with high probability, then the hyperplane conjecture is true.

Going in the same direction, Klartag proved in [17] that every non-degenerate $n$-dimensional measure has one direction that behaves in a "supergaussian way" for $t$ in the interval $1 \leq t \leq c(\log n)^{\frac{1}{4}}$. As a consequence of the aforementioned central limit theorem this interval was extended to $1 \leq t \leq n^{\kappa}$ for some constant $\kappa$ (see 33 ).

The approach used to prove the central limit theorem, as well as some previous weaker results (see [2], 5] or [33]), involved the study of the average of the distribution functions of the 1-dimensional marginals together with a concentration of measure phenomenon. More precisely, Sodin proved that if the Euclidean norm verifies a concentration hypothesis, then the average of the distribution function of the 1-dimensional marginals of an isotropic random vector is approximately Gaussian for $|t| \leq n^{\kappa}$ for some absolute constant $\kappa$, which is smaller than $\frac{1}{4}$ (due to "spherical approximation"). A similar result was obtained for $k$-dimensional marginals in [4. In [19, the concentration hypothesis was shown to be true for every convex body.

In [1, a new approach to study the expected value of the support function of a random polytope generated by $N$ vertices in an isotropic convex body was introduced. Using this approach, we will prove in Section 4 that a supergaussian estimate for the average of the distribution function of the 1-dimensional marginals of the uniform probability measure on an isotropic body holds for the whole range $1 \leq t \leq \frac{\sqrt{n}}{c}$, and not only if $t \leq n^{\kappa}$. Namely, if we define

$$
F(t)=\int_{S^{n-1}}\left|\left\{x \in K:|\langle x, \theta\rangle| \geq t L_{K}\right\}\right| d \sigma(\theta),
$$

where $d \sigma$ denotes the uniform probability measure on $S^{n-1}$, we have the following:

Theorem 1.2. There exists an absolute constant $c$ such that for every symmetric isotropic convex body $K \subseteq \mathbb{R}^{n}$ and every $1 \leq t \leq \frac{\sqrt{n}}{c}$ we have

$$
F(t) \geq e^{-c^{2} t^{2}} .
$$

Using the same idea, in Section 5 we will also show a subgaussian estimate for the average distribution function. However, in this case the estimate does not cover the whole range $1 \leq t \leq c \sqrt{n}$ except for convex bodies with small diameter, i.e., $R(K) \leq C \sqrt{n} L_{K}$.

Theorem 1.3. There exists an absolute constant $c$ such that for every symmetric isotropic convex body $K \subseteq \mathbb{R}^{n}$ and any $1 \leq t \leq n^{\frac{1}{4}}$

$$
F(t) \leq e^{-c^{2} t^{2}} .
$$

Furthermore, if $K$ has small diameter, then this estimate is true for $1 \leq t \leq C \sqrt{n}$.

As a consequence, we will find an interval in which a random direction $\theta \in S^{n-1}$ verifies a subgaussian estimate with high probability. A restriction of the interval leads to better estimates for the measure of the set of "subgaussian directions" in the case of convex bodies with small diameter.

At this point we would also like to mention the paper [6] by Bobkov and Koldobsky, where they studied the rate of convergence of the average volume of sections of an isotropic convex body $K$ to the gaussian density on the line with variance $L_{K}^{2}$, instead of the average of the distribution functions of the 1-dimensional marginals of the uniform probability measure on an isotropic body. 


\section{Preliminaries AND NOTATion}

Before we go into more detail we start with some basic definitions. A convex body $K \subset \mathbb{R}^{n}$ is a compact convex set with non-empty interior. It is called symmetric if $-x \in K$, whenever $x \in K$. We will denote its volume (or Lebesgue measure) by $|\cdot|$. The volume of the Euclidean unit ball $B_{2}^{n}$ will be denoted by $w_{n}=\left|B_{2}^{n}\right|$. We write $S^{n-1}=\left\{x \in \mathbb{R}^{n}:\|x\|_{2}=1\right\}$ for the standard Euclidean sphere in $\mathbb{R}^{n}$ and $d \sigma$ for the uniform probability measure on $S^{n-1}$. A convex body is said to be isotropic if it has volume 1 and satisfies the following two conditions:

- $\int_{K} x d x=0$ (center of mass at 0 ),

- $\int_{K}\langle x, \theta\rangle^{2} d x=L_{K}^{2} \quad \forall \theta \in S^{n-1}$,

where $L_{K}$ is a constant independent of $\theta$, which is called the isotropic constant of $K$. Here, $\langle\cdot, \cdot\rangle$ denotes the standard scalar product in $\mathbb{R}^{n}$.

A probability measure $\mu$ on $\mathbb{R}^{n}$ is said to be isotropic if it is centered at 0 and its covariance matrix is the identity. Notice that a convex body is isotropic if and only if the uniform probability measure on $\frac{K}{L_{K}}$ is isotropic.

Let $K$ be a convex body and $\theta \in S^{n-1}$ a unit vector. The support function of $K$ in the direction $\theta$ is defined by $h_{K}(\theta)=\max \{\langle x, \theta\rangle: x \in K\}$. The mean width of $K$ is

$$
w(K)=\int_{S^{n-1}} h_{K}(\theta) d \sigma(\theta) .
$$

Given a symmetric isotropic convex body $K$, we denote by $K_{N}=\operatorname{conv}\left\{ \pm X_{1}, \ldots\right.$, $\left.\pm X_{N}\right\}$ the random polytope, where $X_{1}, \ldots, X_{N}$ are independent random vectors uniformly distributed in $K$.

In the sequel, if $\mu$ is an isotropic probability measure on $\mathbb{R}^{n}, f_{\theta}$ will denote the density of the random variable $\langle X, \theta\rangle$ with $X$ distributed according to $\mu$. $\gamma$ will denote the density of a standard Gaussian, i.e.,

$$
\gamma(t)=\frac{1}{\sqrt{2 \pi}} e^{-\frac{t^{2}}{2}}
$$

The following lemma is very well known:

Lemma 2.1. For every $t \geq 1$

$$
\frac{\gamma(t)}{2 t} \leq \int_{t}^{\infty} \gamma(s) d s \leq \frac{\gamma(t)}{t}
$$

The letters $c, c^{\prime}, C, C^{\prime}, c_{1}, c_{2}, \ldots$ will denote positive absolute constants, whose value may change from line to line.

Now, let us mention the central limit theorem in the form we will use it to prove Theorem 1.1 in Section 3 ,

Klartag's central limit theorem for isotropic measures, combined with an argument by Sodin 33 , gives the following:

Theorem 2.2 ([19, Theorem 1.4]). Let $n \geq 1$ be an integer and let $X$ be a random vector in $\mathbb{R}^{n}$ with an isotropic, log-concave density. Then, there exists $\Theta \subseteq S^{n-1}$ 
with $\sigma_{n-1}(\Theta) \geq 1-C e^{-\sqrt{n}}$ such that for all $\theta \in \Theta$, the real valued random variable $\langle X, \theta\rangle$ has a density $f_{\theta}: \mathbb{R}^{n} \rightarrow[0, \infty)$ with the following properties:

(1) $\int_{-\infty}^{\infty}\left|f_{\theta}(t)-\gamma(t)\right| d t \leq \frac{1}{n^{\kappa}}$,

(2) For all $|t| \leq n^{\kappa}$ we have $\left|\frac{f_{\theta}(t)}{\gamma(t)}-1\right| \leq \frac{1}{n^{\kappa}}$.

Here, $C, \kappa>0$ are universal constants.

In the case of symmetric $X$, those results give $\kappa=\frac{1}{24}$, obtaining that there exists $\Theta \subseteq S^{n-1}$ with $\sigma_{n-1}(\Theta) \geq 1-C e^{-\sqrt{n}}$ such that for any $\theta \in \Theta$,

$$
\left|\frac{f_{\theta}(t)}{\gamma(t)}-1\right| \leq \frac{C^{\prime}}{n^{\frac{1}{24}}}
$$

whenever $|t|<c n^{\frac{1}{24}}$.

Let us also introduce some notation and results we will need to prove the estimates for the average of the distribution functions of the 1-dimensional marginals.

A convex function $M:[0, \infty) \rightarrow[0, \infty)$ with $M(0)=0$ and $M(t)>0$ for $t>0$ is called an Orlicz function (see for instance [21] or [31]).

Let $X$ be a random vector in $\mathbb{R}^{n}$. For every $\theta \in S^{n-1}$ we define an Orlicz function $M_{\theta}$ by

$$
M_{\theta}(s)=\int_{0}^{s} \int_{\left\{\frac{1}{t} \leq|\langle X, \theta\rangle|\right\}}|\langle X, \theta\rangle| d \mathbb{P} d t .
$$

This Orlicz function was used in [1] to study the expected value of the support function of a random polytope in the direction $\theta$ since if $M_{\theta}$ is the Orlicz function associated to a random vector uniformly distributed on an isotropic body $K$ and $K_{N}$ is a random polytope on $K$, then we have (see [1], Corollary 2.2 )

$$
\mathbb{E} h_{K_{N}}(\theta) \sim \inf \left\{s>0: M_{\theta}\left(\frac{1}{s}\right) \leq \frac{1}{N}\right\} .
$$

The following proposition was obtained:

Proposition 2.3 ([1, Proposition 4.3]). Let $K$ be a symmetric convex body in $\mathbb{R}^{n}$ of volume 1. Let $s>0, \theta \in S^{n-1}$ and $M_{\theta}$ be the Orlicz function associated to the random variable $\langle X, \theta\rangle$, where $X$ is uniformly distributed in $K$. Then,

$$
\int_{S^{n-1}} M_{\theta}\left(\frac{1}{s}\right) d \sigma(\theta)=\int_{K} M_{\left\langle\theta, e_{1}\right\rangle}\left(\frac{\|x\|_{2}}{s}\right) d x,
$$

where $M_{\left\langle\theta, e_{1}\right\rangle}$ is the Orlicz function associated to the random variable $\left\langle\theta, e_{1}\right\rangle$ with $\theta$ uniformly distributed on $S^{n-1}$. For any $s \leq\|x\|_{2}$

$$
M_{\left\langle\theta, e_{1}\right\rangle}\left(\frac{\|x\|_{2}}{s}\right)=\frac{2 w_{n-1}}{n w_{n}} \int_{0}^{\cos ^{-1}\left(\frac{s}{\|x\|_{2}}\right)} \frac{\sin ^{n} y}{\cos ^{2} y} d y,
$$

and 0 otherwise.

With this representation the existence of some directions for which $\mathbb{E} h_{K_{N}}(\theta) \geq$ $C \sqrt{\log N} L_{K}$ holds was established. Using this very same approach we are going to prove Theorem 1.2 and Theorem 1.3 in Section 4 . 


\section{Expected value of the mean width of a Random polytope}

In this section we are going to prove Theorem 1.1 It is a direct consequence of the following theorem, which will fill the aforementioned "gap" in the range of $n$ in the results already proved in 12 .

Theorem 3.1. Let $K$ be a symmetric isotropic convex body and $K_{N}$ a random polytope in $K$, with $n \leq N \leq n^{\delta}$. There exist absolute constants $c, C$ and a set $\Theta \subseteq S^{n-1}$ with $\sigma(\Theta) \geq 1-C e^{-\sqrt{n}}$ such that for every $\theta \in \Theta$

$$
\mathbb{E} h_{K_{N}}(\theta) \geq \frac{c}{\sqrt{\delta}} \sqrt{\log N} L_{K}
$$

Proof. First of all, notice that for every $\theta \in S^{n-1}$

$$
\begin{aligned}
M_{\theta}\left(\frac{1}{s}\right) & =\int_{0}^{\frac{1}{s}} \int_{K \cap\left\{|\langle x, \theta\rangle| \geq \frac{1}{t}\right\}}|\langle x, \theta\rangle| d x d t \\
& \geq \int_{\frac{1}{2 s}}^{\frac{1}{s}} \frac{1}{t}\left|\left\{x \in K:|\langle x, \theta\rangle| \geq \frac{1}{t}\right\}\right| d t \\
& \geq\left(\frac{1}{s}-\frac{1}{2 s}\right) s|\{x \in K:|\langle x, \theta\rangle| \geq 2 s\}| \\
& =\frac{1}{2}|\{x \in K:|\langle x, \theta\rangle| \geq 2 s\}| .
\end{aligned}
$$

Thus, if $s_{0}=t_{0} L_{K}$ such that $M_{\theta}\left(\frac{1}{s_{0}}\right)=\frac{1}{N}$, we have that

$$
\frac{2}{N} \geq\left|\left\{x \in K:|\langle x, \theta\rangle| \geq 2 t_{0} L_{K}\right\}\right|=\mathbb{P}\left\{|\langle Y, \theta\rangle| \geq 2 t_{0}\right\},
$$

where $Y$ is a random variable distributed uniformly on $\frac{K}{L_{K}}$. Thus, if for some $t$ we have $\mathbb{P}\{|\langle Y, \theta\rangle| \geq t\}>\frac{2}{N}$, then $t_{0} \geq \frac{t}{2}$. At this point we would like to mention that one can obtain the same conclusion without using the Orlicz function $M_{\theta}$. However, we decided to do it this way, since we will also use this estimate in the proof of Theorem 1.3. From (2.1) we have that if $Y$ is a log-concave isotropic (covariance matrix equals the identity) random vector in $\mathbb{R}^{n}$, then there exists a subset $\Theta \subseteq S^{n-1}$ with measure greater than $1-C e^{-\sqrt{n}}$ such that for any $\theta \in \Theta$,

$$
\left|\frac{f_{\theta}(t)}{\gamma(t)}-1\right| \leq \frac{C^{\prime}}{n^{\frac{1}{24}}} \text { when }|t| \leq c n^{\frac{1}{24}}
$$

Applying this result to the uniform probability measure on $\frac{K}{L_{K}}$, we have that there exists $\Theta \subseteq S^{n-1}$ with measure greater than $1-C e^{-\sqrt{n}}$ such that for any $\theta \in \Theta$ and ant $0 \leq t \leq c n^{\frac{1}{24}}$ we have, using Lemma 2.1,

$$
\begin{aligned}
\mathbb{P}\{|\langle Y, \theta\rangle|<t\} & \leq\left(1+\frac{C^{\prime}}{n^{\frac{1}{24}}}\right)\left(1-2 \int_{t}^{\infty} \gamma(s) d s\right) \\
& \leq\left(1+\frac{C^{\prime}}{n^{\frac{1}{24}}}\right)\left(1-\frac{\gamma(t)}{t}\right),
\end{aligned}
$$

and so

$$
\mathbb{P}\{|\langle Y, \theta\rangle| \geq t\} \geq 1-\left(1+\frac{C^{\prime}}{n^{\frac{1}{24}}}\right)\left(1-\frac{\gamma(t)}{t}\right) .
$$


Taking $t=\alpha \sqrt{\log N}$ we have that $t \leq c n^{\frac{1}{24}}$, since $N \leq n^{\delta}$. Thus,

$$
\begin{aligned}
\mathbb{P}\{|\langle Y, \theta\rangle| \geq \alpha \sqrt{\log N}\} & \geq \frac{e^{-\frac{\alpha^{2}}{2} \log N}}{\sqrt{2 \pi} \alpha \sqrt{\log N}}-\frac{C^{\prime}}{n^{\frac{1}{24}}}\left(1-\frac{e^{-\frac{\alpha^{2}}{2} \log N}}{\sqrt{2 \pi} \alpha \sqrt{\log N}}\right) \\
& \geq \frac{1}{\sqrt{2 \pi} \alpha N^{\frac{\alpha^{2}}{2}} \sqrt{\log N}}-\frac{C^{\prime}}{n^{\frac{1}{24}}} \\
& \geq \frac{1}{\sqrt{2 \pi} \alpha N^{\frac{\alpha^{2}}{2}} \sqrt{\log N}}-\frac{C^{\prime}}{N^{\frac{1}{24 \delta}}}>\frac{2}{N}
\end{aligned}
$$

whenever $N \geq N_{0}$ if we take $\alpha^{2}=\frac{1}{24 \delta}$. Thus, for every $\theta \in \Theta$,

$$
\mathbb{E} h_{K_{N}}(\theta) \sim s_{0}>\frac{1}{2 \sqrt{24 \delta}} \sqrt{\log N} L_{K} .
$$

\section{Supergaussian estimates}

In this section we use the technique introduced in [1] to prove Theorem [1.2, extending the interval in which the average of the distribution function behaves in a supergaussian way.

Proof of Theorem 1.2. First of all, notice that for any $s>0$ and any isotropic convex body $K$ we have

$$
\begin{aligned}
\int_{S^{n-1}} M_{\theta}\left(\frac{1}{s}\right) d \sigma(\theta) & =\int_{S^{n-1}} \int_{0}^{\frac{1}{s}} \int_{K \cap\left\{|\langle x, \theta\rangle| \geq \frac{1}{t}\right\}}|\langle x, \theta\rangle| d x d t d \sigma(\theta) \\
& \leq \int_{S^{n-1}} \frac{1}{s} \int_{K \cap\{|\langle x, \theta\rangle| \geq s\}}|\langle x, \theta\rangle| d x d \sigma(\theta) \\
& \leq \int_{S^{n-1}} \frac{1}{s} L_{K}|\{x \in K:|\langle x, \theta\rangle| \geq s\}|^{\frac{1}{2}} d \sigma(\theta) \\
& \leq \frac{L_{K}}{s}\left(\int_{S^{n-1}}|\{x \in K:|\langle x, \theta\rangle| \geq s\}| d \sigma(\theta)\right)^{\frac{1}{2}} .
\end{aligned}
$$

Thus, taking $s=t L_{K}$, we have that for any $t>0$,

$$
\left(\int_{S^{n-1}}\left|\left\{x \in K:|\langle x, \theta\rangle| \geq t L_{K}\right\}\right| d \sigma(\theta)\right)^{\frac{1}{2}} \geq t \int_{S^{n-1}} M_{\theta}\left(\frac{1}{t L_{K}}\right) d \sigma(\theta) .
$$

Using the representation of the average of $M_{\theta}$ as an integral on $K$ (Proposition 2.3) and the lower bound obtained in the proof of Theorem 4.2 in [1, we obtain that for every positive $t$,

$$
t \int_{S^{n-1}} M_{\theta}\left(\frac{1}{t L_{K}}\right) d \sigma(\theta) \geq t \int_{K \backslash 2 t L_{K} B_{2}^{n}} \frac{c w_{n-1}}{n w_{n}} \frac{\|x\|_{2}}{t L_{K}} e^{-\frac{C n t^{2} L_{K}^{2}}{\|x\|_{2}^{2}}} d x .
$$

Using the small ball probability estimate from [25], there exists an absolute constant $c_{1}$ such that $\left|K \backslash c_{1} \sqrt{n} L_{K}\right| \geq \frac{1}{2}$. Thus, if $0<t \leq \frac{c_{1}}{2} \sqrt{n}$,

$$
\begin{aligned}
\left(\int_{S^{n-1}}\left|\left\{x \in K:|\langle x, \theta\rangle|>t L_{K}\right\}\right| d \sigma(\theta)\right)^{\frac{1}{2}} & \geq \int_{K \backslash c_{1} \sqrt{n} L_{K} B_{2}^{n}} \frac{c w_{n-1}}{n w_{n}} \frac{\|x\|_{2}}{L_{K}} e^{-\frac{C n t^{2} L_{K}^{2}}{\|x\|_{2}^{2}}} d x \\
& \geq c_{2} e^{-C t^{2}} \geq e^{-c_{3} t^{2}}
\end{aligned}
$$


if $1 \leq t \leq \frac{c_{1}}{2} \sqrt{n}$. Thus, taking $c=\max \left\{\frac{2}{c_{1}}, 2 c_{3}\right\}$, we obtain that for every isotropic convex body $K$ and every $1 \leq t \leq \frac{\sqrt{n}}{c}$,

$$
\int_{S^{n-1}}\left|\left\{x \in K:|\langle x, \theta\rangle|>t L_{K}\right\}\right| d \sigma(\theta) \geq e^{-c t^{2}} .
$$

As a consequence, we obtain the following estimate of the measure of the set of directions verifying a supergaussian estimate for a particular $t$. However, opposite to what will happen for a subgaussian estimate, the estimate of the measure of this set of directions will be very small for big values of $t$.

Corollary 4.1. There exist absolute constants $c, c^{\prime}$ such that for every isotropic symmetric convex body $K \subseteq \mathbb{R}^{n}$ and for every $1 \leq t \leq \frac{\sqrt{n}}{c}$, the set of directions verifying the supergaussian estimate

$$
\left|\left\{x \in K:|\langle x, \theta\rangle| \geq t L_{K}\right\}\right| \geq e^{-c^{2} t^{2}}
$$

has measure greater than $e^{-c^{\prime} t^{2}}$.

Proof. By Theorem 1.2

$$
\int_{S^{n-1}}\left|\left\{x \in K:|\langle x, \theta\rangle|<t L_{K}\right\}\right| d \sigma(\theta) \leq 1-e^{-c^{2} t^{2}} .
$$

Thus, by Markov's inequality, we have

$$
\sigma\left\{\theta \in S^{n-1}:\left|\left\{x \in K:|\langle x, \theta\rangle|<t L_{K}\right\}\right|>1-e^{-c^{\prime 2} t^{2}}\right\} \leq \frac{1-e^{-c^{2} t^{2}}}{1-e^{-c^{\prime 2} t^{2}}} .
$$

Consequently,

$$
\sigma\left\{\theta \in S^{n-1}:\left|\left\{x \in K:|\langle x, \theta\rangle| \geq t L_{K}\right\}\right|<e^{-c^{\prime 2} t^{2}}\right\} \leq \frac{1-e^{-c^{2} t^{2}}}{1-e^{-c^{\prime 2} t^{2}}},
$$

and so

$$
\begin{aligned}
\sigma\left\{\theta \in S^{n-1}:\left|\left\{x \in K:|\langle x, \theta\rangle| \geq t L_{K}\right\}\right| \geq e^{-c^{\prime 2} t^{2}}\right\} & \geq 1-\frac{1-e^{-c^{2} t^{2}}}{1-e^{-c^{\prime 2} t^{2}}} \\
& =\frac{e^{-c^{2} t^{2}}\left(1-e^{-\left(c^{\prime 2}-c^{2}\right) t^{2}}\right)}{1-e^{-c^{\prime 2} t^{2}}} .
\end{aligned}
$$

Choosing ${c^{\prime}}^{2}=2 c^{2}$ we obtain the result.

\section{Subgaussian estimates}

In this section we will extend the interval in which the average of the distribution function verifies a subgaussian estimate. As a consequence, we will obtain an estimate of the measure of the directions verifying a subgaussian estimate in some interval.

Proof of Theorem 1.3. As we have seen in the proof of Theorem 1.1 for every $\theta \in$ $S^{n-1}$, we have

$$
M_{\theta}\left(\frac{1}{s}\right) \geq \frac{1}{2}|\{x \in K:|\langle x, \theta\rangle| \geq 2 s\}| .
$$

Thus, using the upper bound shown in 1

$$
F(t) \leq 2 \int_{S^{n-1}} M_{\theta}\left(\frac{2}{t L_{K}}\right) d \sigma(\theta) \leq \frac{4 \omega_{n-1}}{n \omega_{n} t L_{K}} \int_{K}|x| e^{-\frac{(n-1) t^{2} L_{K}^{2}}{2|x|^{2}}} d x .
$$


By Paouris' concentration of measure result, there exist constants such that for every $\gamma>c_{1},\left|K \backslash \gamma \sqrt{n} L_{K} B_{2}^{n}\right| \leq e^{-c_{2} \gamma \sqrt{n}}$. Hence,

$$
\begin{aligned}
\int_{K}|x| e^{-\frac{(n-1) t^{2} L_{K}^{2}}{2|x|^{2}}} d x & =\int_{K \cap \gamma \sqrt{n} L_{K} B_{2}^{n}}|x| e^{-\frac{(n-1) t^{2} L_{K}^{2}}{2|x|^{2}}} d x+\int_{K \backslash \gamma \sqrt{n} L_{K} B_{2}^{n}}|x| e^{-\frac{(n-1) t^{2} L_{K}^{2}}{2|x|^{2}}} d x \\
& \leq \gamma \sqrt{n} L_{K} e^{-\frac{c t^{2}}{\gamma^{2}}}+(n+1) L_{K}\left|K \backslash \gamma \sqrt{n} L_{K} B_{2}^{n}\right| \\
& \leq \gamma \sqrt{n} L_{K} e^{-\frac{c t^{2}}{\gamma^{2}}}+(n+1) L_{K} e^{-c_{2} \gamma \sqrt{n}} .
\end{aligned}
$$

Consequently,

$$
\begin{aligned}
F(t) & \leq \frac{C}{t}\left(\gamma e^{-\frac{c t^{2}}{\gamma^{2}}}+\sqrt{n} e^{-c_{2} \gamma \sqrt{n}}\right) \\
& \leq \frac{C}{t}\left(\gamma e^{-\frac{c t^{2}}{\gamma^{2}}}+e^{\sqrt{n}} e^{-c_{2} \gamma \sqrt{n}}\right) \\
& \leq \frac{C}{t}\left(\gamma e^{-\frac{c t^{2}}{\gamma^{2}}}+e^{\left(1-c_{2} \gamma\right) t^{2}}\right)
\end{aligned}
$$

if $1 \leq t \leq n^{\frac{1}{4}}$ and $\gamma$ is a constant big enough. Thus, we obtain that for $t$ in the aforementioned interval

$$
F(t) \leq \frac{C}{t} e^{-c t^{2}} \leq e^{-c^{\prime} t^{2}}
$$

Notice that if $R(K) \leq C \sqrt{n} L_{K}$, then we do not need to split the integral as the sum of two integrals and we obtain that

$$
F(t) \leq \frac{C}{t} e^{-c t^{2}} \leq e^{-c^{\prime} t^{2}}
$$

for every $1 \leq t \leq C \sqrt{n}$.

As a consequence we will obtain that if we consider an interval $t_{0} \leq t \leq n^{\frac{1}{4}}$ with $t_{0} \mathrm{big}$, then the measure of the directions that are subgaussian in such an interval will be big. It is explicitly stated in the following corollary.

Corollary 5.1. Let $K \subseteq \mathbb{R}^{n}$ be an isotropic symmetric convex body and let $1 \leq$ $t_{0} \leq n^{\frac{1}{4}}$. Then, the set of directions $\theta \in S^{n-1}$ that verify

$$
\left|\left\{x \in K:|\langle x, \theta\rangle| \geq t L_{K}\right\}\right| \leq e^{-c^{2} t^{2}} \text { for every } t_{0} \leq t \leq n^{\frac{1}{4}}
$$

has measure greater than $1-e^{-c^{2} t_{0}^{2}}$.

Furthermore, if $K$ has small diameter, we can take $t_{0} \leq C \sqrt{n}$ and $t_{0} \leq t \leq C \sqrt{n}$.

Proof. Applying Markov's inequality in Theorem 1.3, we have that for every $1 \leq$ $t \leq n^{\frac{1}{4}}$

$$
\sigma\left\{\theta \in S^{n-1}: \mid\left\{x \in K:|\langle x, \theta\rangle| \geq t L_{K}\right\} \geq e^{-c^{\prime 2} t^{2}}\right\} \leq e^{-\left(c^{2}-c^{\prime 2}\right) t^{2}} .
$$

Taking $c^{\prime 2}=\frac{1}{2} c^{2}$ we have that there exists a constant $c_{0}$ such that for every $1 \leq t \leq n^{\frac{1}{4}}$

$$
\sigma\left\{\theta \in S^{n-1}:\left|\left\{x \in K:|\langle x, \theta\rangle| \geq t L_{K}\right\}\right| \geq e^{-c_{0}^{2} t^{2}}\right\} \leq e^{-c_{0}^{2} t^{2}} .
$$

Now take $t_{0}<t_{1}<\cdots<t_{I}=n^{\frac{1}{4}}$. Then,

$$
\sigma\left\{\theta \in S^{n-1}: \exists 0 \leq i \leq I \text { with }\left|\left\{x \in K:|\langle x, \theta\rangle| \geq t_{i} L_{K}\right\}\right| \geq e^{-c_{0}^{2} t_{i}^{2}}\right\} \leq \sum_{i=0}^{I} e^{-c_{0}^{2} t_{i}^{2}}
$$


Taking $t_{i}^{2}=t_{0}^{2}+\lambda i$, this probability is bounded by $e^{-c_{0}^{2} t_{0}^{2}} \frac{1}{1-e^{-c_{0}^{2} \lambda}}$. If for every $i$ we have

$$
\left|\left\{x \in K:|\langle x, \theta\rangle| \geq t_{i} L_{K}\right\}\right| \leq e^{-c_{0}^{2} t_{i}^{2}},
$$

then for every $t_{i} \leq t \leq t_{i+1}$ we have

$$
\begin{aligned}
\left|\left\{x \in K:|\langle x, \theta\rangle| \geq t L_{K}\right\}\right| & \leq\left|\left\{x \in K:|\langle x, \theta\rangle| \geq t_{i} L_{K}\right\}\right| \leq e^{-c_{0}{ }^{2} t_{i}^{2}} \\
& =e^{-c_{0}{ }^{2} t^{2}} e^{c_{0}{ }^{2}\left(t^{2}-t_{i}^{2}\right)} \leq e^{-c_{0}{ }^{2} t^{2}} e^{c_{0}{ }^{2}\left(t_{i+1}^{2}-t_{i}^{2}\right)} \\
& =e^{-c_{0}{ }^{2} t^{2}} e^{c_{0}{ }^{2} \lambda}
\end{aligned}
$$

Choosing $\lambda$, a constant smaller than 1 , we obtain the result.

If $K$ has small diameter, the same proof works in the interval $t_{0} \leq t \leq C \sqrt{n}$ with $1 \leq t_{0} \leq C \sqrt{n}$.

\section{ACKNOWLEDGEMENTS}

This work was done while the authors were postdoctoral fellows at the Department of Mathematical and Statistical Sciences at the University of Alberta. They would like to thank the department for providing such a good environment and working conditions. They would also like to thank their colleagues Jesus Bastero, Alexander Litvak and Peter Pivovarov for helpful comments and for reading a preliminary version of this paper.

\section{REFERENCES}

[1] David Alonso-Gutiérrez and Joscha Prochno, Estimating support functions of random polytopes via Orlicz norms, Discrete Comput. Geom. 49 (2013), no. 3, 558-588, DOI 10.1007/s00454-012-9468-7. MR 3038530

[2] Milla Anttila, Keith Ball, and Irini Perissinaki, The central limit problem for convex bodies, Trans. Amer. Math. Soc. 355 (2003), no. 12, 4723-4735 (electronic), DOI 10.1090/S00029947-03-03085-X. MR.1997580 (2005b:52010)

[3] Imre Bárány, Random polytopes, convex bodies, and approximation, Stochastic geometry, Lecture Notes in Math., vol. 1892, Springer, Berlin, 2007, pp. 77-118, DOI 10.1007/978-3540-38175-4_2. MR2327291 (2008e:60026)

[4] Jesús Bastero and Julio Bernués, Asymptotic behaviour of averages of $k$-dimensional marginals of measures on $\mathbb{R}^{n}$, Studia Math. 190 (2009), no. 1, 1-31, DOI 10.4064/sm190-1-1. MR.2457285 (2010b:52004)

[5] S. G. Bobkov, On concentration of distributions of random weighted sums, Ann. Probab. 31 (2003), no. 1, 195-215, DOI 10.1214/aop/1046294309. MR1959791 (2004a:60049)

[6] Sergey G. Bobkov and Alexander Koldobsky, On the central limit property of convex bodies, Geometric aspects of functional analysis, Lecture Notes in Math., vol. 1807, Springer, Berlin, 2003, pp. 44-52, DOI 10.1007/978-3-540-36428-3_5. MR2083387 (2005j:60044)

[7] Sergey G. Bobkov and Fedor L. Nazarov, On convex bodies and log-concave probability measures with unconditional basis, Geometric aspects of functional analysis, Lecture Notes in Math., vol. 1807, Berlin, 2003, pp. 53-69, DOI 10.1007/978-3-540-36428-3_6. MR2083388 (2005k:60058)

[8] Sergey G. Bobkov and Fedor L. Nazarov, Large deviations of typical linear functionals on a convex body with unconditional basis, Stochastic inequalities and applications, Progr. Probab., vol. 56, Birkhäuser, Basel, 2003, pp. 3-13. MR2073422 (2005f:52013)

[9] J. Bourgain, On high-dimensional maximal functions associated to convex bodies, Amer. J. Math. 108 (1986), no. 6, 1467-1476, DOI 10.2307/2374532. MR868898(88h:42020)

[10] Jean Bourgain, On the isotropy-constant problem for "PSI-2"-bodies, Geometric aspects of functional analysis, Lecture Notes in Math., vol. 1807, Springer, Berlin, 2003, pp. 114-121, DOI 10.1007/978-3-540-36428-3_9. MR2083391 (2006c:46011) 
[11] N. Dafnis, A. Giannopoulos, and A. Tsolomitis, Asymptotic shape of a random polytope in a convex body, J. Funct. Anal. 257 (2009), no. 9, 2820-2839, DOI 10.1016/j.jfa.2009.06.027. MR.2559718(2011b:52008)

[12] N. Dafnis, A. Giannopoulos, and A. Tsolomitis, Quermaßintegrals and asymptotic shape of random polytopes in an isotropic convex body, Michigan Math. J. 62 (2013), no. 1, 59-79, DOI 10.1307/mmj/1363958241. MR3049297

[13] A. Giannopoulos, G. Paouris, and P. Valettas, On the existence of subgaussian directions for log-concave measures, Concentration, functional inequalities and isoperimetry, Contemp. Math., vol. 545, Amer. Math. Soc., Providence, RI, 2011, pp. 103-122, DOI 10.1090/conm/545/10768. MR2858469 (2012k:52007)

[14] E. D. Gluskin, The diameter of the Minkowski compactum is roughly equal to $n$ (Russian), Funktsional. Anal. i Prilozhen. 15 (1981), no. 1, 72-73. MR609798 (83d:46026)

[15] E. D. Gluskin, A. E. Litvak, and N. Tomczak-Jaegermann, An example of a convex body without symmetric projections, Israel J. Math. 124 (2001), 267-277, DOI 10.1007/BF02772622. MR.1856519 (2003e:52006)

[16] Douglas Hensley, Slicing convex bodies-bounds for slice area in terms of the body's covariance, Proc. Amer. Math. Soc. 79 (1980), no. 4, 619-625, DOI 10.2307/2042510. MR.572315 (81j:52008)

[17] Bo'az Klartag, On nearly radial marginals of high-dimensional probability measures, J. Eur. Math. Soc. (JEMS) 12 (2010), no. 3, 723-754, DOI 10.4171/JEMS/213. MR2639317 (2011d:60008)

[18] B. Klartag, Uniform almost sub-Gaussian estimates for linear functionals on convex sets, Algebra i Analiz 19 (2007), no. 1, 109-148, DOI 10.1090/S1061-0022-07-00987-9; English transl., St. Petersburg Math. J. 19 (2008), no. 1, 77-106. MR2319512(2008f:60018)

[19] B. Klartag, Power-law estimates for the central limit theorem for convex sets, J. Funct. Anal. 245 (2007), no. 1, 284-310, DOI 10.1016/j.jfa.2006.12.005. MR2311626 (2008e:60049)

[20] Bo'az Klartag and Gady Kozma, On the hyperplane conjecture for random convex sets, Israel J. Math. 170 (2009), 253-268, DOI 10.1007/s11856-009-0028-7. MR2506326 (2010c:60031)

[21] M. A. Krasnosel'skiı̌ and Ja. B. Rutickǐ̌, Convex functions and Orlicz spaces, translated from the first Russian edition by Leo F. Boron, P. Noordhoff Ltd., Groningen, 1961. MR0126722 (23 \#A4016)

[22] Vitali D. Milman and Alain Pajor, Isotropic position and inertia ellipsoids and zonoids of the unit ball of a normed $n$-dimensional space, Geometric aspects of functional analysis (1987-88), Lecture Notes in Math. 1376 (1989), Springer, Berlin, pp. 64-104, DOI 10.1007/BFb0090049. MR 1008717 (90g:52003)

[23] Fedor Nazarov, On the maximal perimeter of a convex set in $\mathbb{R}^{n}$ with respect to a Gaussian measure, Geometric aspects of functional analysis, Lecture Notes in Math. 1807 (2003), Springer, Berlin, pp. 169-187, DOI 10.1007/978-3-540-36428-3_15. MR2083397(2005h:28032)

[24] G. Paouris, Concentration of mass on convex bodies, Geom. Funct. Anal. 16 (2006), no. 5, 1021-1049, DOI 10.1007/s00039-006-0584-5. MR2276533 (2007k:52009)

[25] Grigoris Paouris, Small ball probability estimates for log-concave measures, Trans. Amer. Math. Soc. 364 (2012), no. 1, 287-308, DOI 10.1090/S0002-9947-2011-05411-5. MR2833584

[26] Grigoris Paouris, $\Psi_{2}$-estimates for linear functionals on zonoids, Geometric aspects of functional analysis, Lecture Notes in Math. 1807 (2003), Springer, Berlin, pp. 211-222, DOI 10.1007/978-3-540-36428-3_17. MR2083399 (2005g:52021)

[27] G. Paouris, On the $\psi_{2}$-behaviour of linear functionals on isotropic convex bodies, Studia Math. 168 (2005), no. 3, 285-299, DOI 10.4064/sm168-3-7. MR2146128(2006c:52004)

[28] Grigoris Paouris, On the existence of supergaussian directions on convex bodies, Mathematika 58 (2012), no. 2, 389-408, DOI 10.1112/S0025579311006085. MR2965979

[29] Grigoris Paouris and Peter Pivovarov, A probabilistic take on isoperimetric-type inequalities, Adv. Math. 230 (2012), no. 3, 1402-1422, DOI 10.1016/j.aim.2012.03.019. MR2921184

[30] Peter Pivovarov, On the volume of caps and bounding the mean-width of an isotropic convex body, Math. Proc. Cambridge Philos. Soc. 149 (2010), no. 2, 317-331, DOI 10.1017/S0305004110000216. MR2670218 (2012a:52020)

[31] M. M. Rao and Z. D. Ren, Theory of Orlicz spaces, Monographs and Textbooks in Pure and Applied Mathematics, vol. 146, Marcel Dekker Inc., New York, 1991. MR1113700(92e:46059) 
[32] Matthias Reitzner, Random polytopes, New perspectives in stochastic geometry, Oxford Univ. Press, Oxford, 2010, pp. 45-76. MR2654675 (2011g:60026)

[33] S. Sodin, Tail-sensitive Gaussian asymptotics for marginals of concentrated measures in high dimension, Geometric aspects of functional analysis, Lecture Notes in Math. 1910 (2007), Springer, Berlin, pp. 271-295, DOI 10.1007/978-3-540-72053-9_16. MR2349613(2008j:60035)

[34] Stanisław J. Szarek, The finite-dimensional basis problem with an appendix on nets of Grassmann manifolds, Acta Math. 151 (1983), no. 3-4, 153-179, DOI 10.1007/BF02393205. MR723008(85i:47024)

Department of Mathematical and Statistical Sciences, University of Alberta, 505 Central Academic Building, Edmonton T6G 2G1, Canada

Current address: Departament de Matemàtiques, Universitat Jaume I, Campus de Riu Sec, E-12071 Castelló de la Plana, Spain

E-mail address: alonsod@uji.es

Department of Mathematical and Statistical Sciences, University of Alberta, 605 Central Academic Bullding, Edmonton T6G 2G1, Canada

Current address: Institute of Analysis, Johannes Keples University, Liuz Altunbergerstr. 69, 4040 Liuz, Austria

E-mail address: prochno@ualberta.ca 